\title{
Comparison of pretreatment assessment of intrauterine tumor spread in endometrial carcinoma using ultrasonography, hysteroscopy, and fractional curettage
}

\author{
F. C. Thiel • S. P. Renner • S. Ackermann • H. Binder • \\ B. Meurer • M. Schrauder • A. Müller • \\ M. W. Beckmann $\cdot$ P. Oppelt
}

Received: 26 October 2006 / Accepted: 4 July 2007 / Published online: 24 August 2007

(C) Springer-Verlag 2007

\begin{abstract}
Due to their age, patients with endometrial carcinoma are often in an impaired general condition and have other concomitant diseases. To prevent overtreatment or undertreatment, invasive and noninvasive diagnostic procedures such as ultrasonography, hysteroscopy, and fractional curettage were compared with regard to their capacity to assess tumor extent (in) to the cervix. In 75 patients with endometrial carcinoma, the results of transvaginal ultrasonography, diagnostic hysteroscopy, and fractional curettage in assessing tumor spread to the cervix were compared with the final pathology report on the hysterectomy specimen. Cervical involvement was demonstrated in the hysterectomy specimen in $25.3 \%$ of the patients (19 of 75). Ultrasound identified evidence of cervical involvement with a sensitivity of $15.8 \%$ (3 of 19) and a specificity of $98.1 \%$ (53 of 54); hysteroscopy had a sensitivity of $42.9 \%$ (6 of 14 ) and a specificity of $89.5 \%$ (34 of 38); and fractional curettage had a sensitivity of $57.9 \%$ (11 of 19) and a specificity of $66.1 \%$ ( 37 of 56 ). None of the procedures on its own is suitable for pretreatment assessment of cervical involvement. However, negative endocervical curettage and hysteroscopical exclusion of cervical infiltration may often
\end{abstract}

F. C. Thiel $(\bowtie) \cdot S$. P. Renner $\cdot$ S. Ackermann $\cdot H$. Binder $\cdot$ B. Meurer · M. Schrauder · A. Müller · M. W. Beckmann • P. Oppelt

Department of Gynecology, Erlangen University Hospital, Universitätsstrasse 21-23,

91054 Erlangen, Germany

e-mail: falk.thiel@gyn.imed.uni-erlangen.de

M. Schrauder

email: michael.schrauder@gyn.imed.uni-erlangen.de identify patients correctly without cervical involvement, thereby avoiding overtreatment.

Keywords Endometrial carcinoma - Ultrasonography . Hysteroscopy $\cdot$ Fractional dilation and curettage

\section{Introduction}

Endometrial carcinoma is the most frequent type of genital carcinoma among women, with an annual incidence of 24.7 per 100,000 women. With the mean age of onset being 68 , it is mainly a disease of older women, who are usually in an impaired general condition due to concomitant diseases such as hypertension, coronary heart disease, diabetes mellitus, and obesity [1]. Stage-adjusted treatment needs to be carried out in order to avoid increasing the morbidity. Due to the early symptom of postmenopausal bleeding, a majority representing $75 \%$ of patients with the disease are diagnosed in International Federation of Gynecology and Obstetrics (FIGO) stage I. Recent findings have shown that the less invasive laparoscopy-assisted vaginal hysterectomy (LAVH) operation, with bilateral adnexectomy and with laparoscopic lymphadenectomy if appropriate, is a reliable surgical method for the treatment of FIGO stage I endometrial carcinoma [2].

A 5-year survival rate of almost $90 \%$ is achieved in patients in FIGO stage I. This differs markedly from FIGO stage II, found in $10-20 \%$ of the patients, in which there is infiltration of the cervix. In this stage, the 5-year survival rate is in the range of $60-70 \%$. When there is tumor infiltration as far as the cervical stroma (FIGO IIB), the National Comprehensive Cancer Network (NCCN) in the 
USA and the Gynecological Oncology Study Group (Arbeitsgemeinschaft Gynäkologische Onkologie, AGO) in Germany recommend radical hysterectomy with bilateral adnexectomy and pelvic and para-aortic lymphadenectomy [3-5]. These recommendations and guidelines are important not only for medicolegal reasons, but also in providing patients with an acceptable quality of care [6]. The background to such recommendations is that there are still substantial differences between various gynecology departments with regard to the type of treatment provided for gynecological tumors [7]. Particularly in older patients with multiple concomitant diseases, undertreatment and especially overtreatment need to be avoided. It appears all the more important to be able to assess the tumor involvement of the cervix even before treatment. Various simple and minimally invasive methods are available for this, such as transvaginal ultrasonography, hysteroscopy, and fractional curettage.

The aim of the present study was therefore to assess the diagnostic yield of the individual procedures in endometrial carcinoma with regard to tumor spread to the cervix.

\section{Materials and methods}

Between January 2003 and December 2005, 75 patients were referred to the Department of Gynecology at Erlangen University Hospital due to postmenopausal bleeding or sonographically suspicious endometrium and received initial treatment for endometrial carcinoma. The patients' average age was 65.6 years, with a range of 33-90 years. Twenty-three patients $(30.7 \%)$ had already undergone curettage elsewhere with private gynecologists, without hysteroscopy. A routine ultrasound examination of the uterus and adnexa was carried out in all of the 75 patients. The remaining 52 patients $(69.3 \%)$ underwent our hospital's standard procedure of hysteroscopy and curettage to diagnose an endometrial carcinoma. Due to their diagnoses, all of the 75 patients underwent surgery in accordance with their clinical stage and general condition.

For pretreatment assessment of the extent of tumor spread to the cervix, the patients' ultrasound findings were evaluated in relation to cervical infiltration. Tumor involvement of the cervix was considered to be present if the craniocaudal extension of the educible tumor reached the isthmo-cervical area, also if the endometrium of the cervix shows a poorly definable border to the myometrium, an increased vascular perfusion, an inhomogeneous structure, or an increased endometrial thickness.

Hysteroscopy reports were used to establish the macroscopic location and extent of the carcinoma (in) to the cervix. The hysteroscopy was always performed before curettage.
The histological findings from the fractional curettage were examined for the presence of tumor cells in the cervical abrasion samples. First, the cervical curettage was performed separately, and then the curettage of the uterine cavity. Generally, cervical infiltration can only be unequivocally confirmed in cervical abrasion samples if tumor cells are found to be infiltrating endocervical glands or the endocervical stroma. Due to the difficulty for the pathologists to detect the cervical infiltration as above defined, a cervical involvement was considered to be present if there was morphological evidence of the carcinoma in the cervical abrasion sample.

Finally, all of the diagnostic data obtained were compared with the ultimate histological findings and the TNM stage after definitive surgical treatment and were analysed statistically using the Statistical Package for the Social Sciences (SPSS) for Windows program, version 12.0 (SPSS, Inc., Chicago, Illinois, USA).

The study was approved by the ethics committee of the department of medicine at Erlangen University Hospital.

\section{Results}

Surgical specimens FIGO stage I endometrial carcinoma was histologically confirmed in $58.7 \%$ of the patients (44 of 75) after definitive surgical treatment; stage II was diagnosed in $14.7 \%$ (11 of 75 ), stage III in $10.7 \%$ (8 of 75 ), and stage IV in $2.7 \%$ ( 2 of 75 ). Particularly, a stage pT2a was found in $6.7 \%$ ( 5 of 75 ) of patients, a stage pT2b in $14.7 \%$ ( 11 of 75 ), and a higher stage than pT2b with involvement of the cervical stroma in $4.0 \%$ (3 of 75 ). According to the histological findings, cervical involvement was present in $25.3 \%$ of the patients (19 of 75 ). In one hysterectomy specimen, only adenomatous endometrial hyperplasia with atypia was found, although endometrial carcinoma had been confirmed in the corpus abrasion sample. Eighty-eight percent of the patients (66 of 75) underwent a simple hysterectomy with bilateral adnexectomy \pm lymphadenectomy, and $12 \%$ of the patients ( 9 of

Table 1 The accuracy, sensitivity, specificity, and positive and negative predictive values of various diagnostic procedures in endometrial carcinoma

\begin{tabular}{llll}
\hline & US (\%) & H (\%) & D\&C (\%) \\
\hline Accuracy & 76.7 & 76.9 & 64.0 \\
Sensitivity & 15.8 & 42.9 & 57.9 \\
Specificity & 98.1 & 89.5 & 66.1 \\
Positive predictive value & 75.0 & 60.0 & 36.7 \\
Negative predictive value & 76.8 & 81.0 & 82.2 \\
\hline
\end{tabular}

$U S$ ultrasonography, $H$ hysteroscopy, $D \& C$ fractional dilation and curettage 
75) underwent radical hysterectomy with bilateral adnexectomy \pm lymphadenectomy.

Transvaginal ultrasonography Transvaginal ultrasonography accurately excluded or confirmed cervical involvement in $76.7 \%$ of the patients ( 56 of 73 ; Table 1 ). There was a sensitivity of $15.8 \%$ (3 of 19), a specificity of $98.1 \%$ (53 of 54 ), a positive predictive value of $75.0 \%$ (3 of 4 ), and a negative predictive value of $76.8 \%$ (53 of 69 ).

Hysteroscopy The extent of tumor spread on the cervix was correctly assessed by hysteroscopy in $76.9 \%$ of the patients (40 of 52). The sensitivity was $42.9 \%$ (6 of 14), the specificity $89.5 \%$ (34 of 38 ), the positive predictive value $60.0 \%$ (6 of 10 ), and the negative predictive value $81.0 \%$ (34 of 42). The sensitivity of hysteroscopy varied depending on the type of cervical involvement. In involvement limited to the cervical glands (pT2a), it was $20.0 \%$ ( 1 in 5), while with infiltration as far as the cervical stroma $(\geq \mathrm{pT} 2 \mathrm{~b})$ it was $55.6 \%$ (5 of 9).

Fractional curettage The sensitivity of fractional curettage was $57.9 \%$ (11 of 19), with a specificity of $66.1 \%$ (37 of 56 ), a positive predictive value of $36.7 \%$ (11 of 30 ), and a negative predictive value of $82.2 \%$ ( 37 of 45 ). Cervical involvement was accurately confirmed or excluded in $64.0 \%$ of the patients ( 48 of 75 ).

In five of the 19 patients with tumor involvement of the cervix in the hysterectomy specimen, the involvement was limited to the cervical glands (pT2a) and had been demonstrated with ultrasonography, hysteroscopy, or curettage in only one case each. The remaining 14 patients had involvement of the cervical stroma ( $\geq \mathrm{pT} 2 \mathrm{~b}$ ). Ultrasonography identified these cases in $14.3 \%$ ( 2 of 14), hysteroscopy in $55.6 \%$ ( 5 of 9 ), and cervical curettage in $71.4 \%$ (10 of 14).

Ten of 14 patients $(71.4 \%)$ with involvement of the cervical stroma $(\geq \mathrm{pT} 2 \mathrm{~b})$ underwent a simple hysterectomy with bilateral adnexectomy \pm lymphadenectomy, and five of 61 patients $(8.2 \%)$ without involvement of the cervical stroma underwent a radical hysterectomy with bilateral adnexectomy \pm lymphadenectomy.

A positive cytology during definitive surgical treatment after hysteroscopy was detected in $1.9 \%$ ( 1 of 52 ).

\section{Discussion}

In accordance with the 1988 FIGO recommendations, the staging of endometrial carcinoma is carried out surgically [8]. The deterioration in the prognosis associated with cervical involvement in endometrial carcinoma was recog- nized as early as 1941 by Heyman et al. [9]. In FIGO stage IIB, the National Comprehensive Cancer Network (NCCN) and the Gynecological Oncology Study Group (Arbeitsgemeinschaft Gynäkologische Onkologie, AGO) therefore currently recommend radical hysterectomy [3-5]. This indicates the importance of preoperative diagnosis of the intracavitary extent of tumor spread. Particularly in older patients with other concomitant diseases, undertreatment and especially overtreatment need to be avoided.

On the basis of their 1990 study including 160 patients who underwent surgery for endometrial carcinoma, Ayhan et al. consider that only preoperative diagnosis of the extent of tumor infiltration into the cervix in endometrial carcinoma is inadequate [10]. Cervical involvement was demonstrated in $12.5 \%$ of the patients who were clinically considered to be in FIGO stage II after endocervical curettage. In $8.6 \%$ of patients with clinical stage $\mathrm{I}$, occult involvement of the cervix was discovered postoperatively. In our own group, fractional curettage showed a false-positive rate as high as $63.3 \%$ and a false-negative rate of $17.8 \%$, with a sensitivity of $57.9 \%$ and a specificity of $66.1 \%$ (Table 2). By contrast, Toki et al., in a study including 64 patients who underwent endocervical curettage, reported a high sensitivity rate of $91 \%$, with a high negative predictive value of $96 \%$ [11]. The greatest sources of error in relation to the high false-positive rates appear to be the spreading of tumor cells into the cervical canal due to instrument manipulation, and intraluminal projection of tumor cones. According to the NCCN and AGO recommendations, cervical infiltration can only be unequivocally confirmed in cervical abrasion samples if tumor cells are found to be infiltrating endocervical glands or the endocervical stroma $[3,4]$. However, such findings are often only diagnosed with difficulty.

Wang and Guo have shown that hysteroscopy is capable of predicting cervical infiltration more accurately than fractional curettage alone [12]. In their study including 156 patients, $90 \%$ of cases of cervical infiltration were correctly demonstrated or excluded by hysteroscopy. This was possible with endocervical curettage in only $75 \%$ of the cases. Mencaglia et al. had already published similar results in 1984 [13], with hysteroscopy showing an accuracy rate of $92 \%$ for excluding or confirming cervical involvement, with no false-negative results and two falsepositive results. However, the study only included 23 patients. In a retrospective study including 200 patients, Lo et al. compared the value of hysteroscopy and intraoperative macroscopic assessment in the diagnosis of cervical involvement [14]. With hysteroscopy, they reported an accuracy of $92.5 \%$, a sensitivity of $68.3 \%$, a specificity of $98.7 \%$, a positive predictive value of $93.3 \%$, and a negative predictive value of $92.4 \%$. In the authors' view, this suggested that hysteroscopy should play a substantial role in the preoperative diagnosis of endometrial carcinoma. 
Table 2 Comparison of studies reporting the accuracy, sensitivity, specificity, and positive and negative predictive values of various diagnostic procedures in endometrial carcinoma

$D \& C$ fractional dilation and curettage, $H$ hysteroscopy, $N P V$ negative predictive value, $P P V$ positive predictive value, US ultrasonography

\begin{tabular}{|c|c|c|c|c|c|c|c|}
\hline $\begin{array}{l}\text { Author(s), year, } \\
\text { reference }\end{array}$ & $\begin{array}{l}\text { Patients } \\
\text { (n) }\end{array}$ & $\begin{array}{l}\text { Diagnostic } \\
\text { procedure (\%) }\end{array}$ & $\begin{array}{l}\text { Accuracy } \\
(\%)\end{array}$ & $\begin{array}{l}\text { Sensitivity } \\
(\%)\end{array}$ & $\begin{array}{l}\text { Specificity } \\
(\%)\end{array}$ & $\begin{array}{l}\text { PPV } \\
(\%)\end{array}$ & $\begin{array}{l}\text { NPV } \\
(\%)\end{array}$ \\
\hline $\begin{array}{l}\text { Ayhan et al. } \\
\text { (1990) [10] }\end{array}$ & 160 & $\mathrm{D} \& \mathrm{C}$ & & & & 87.5 & 91.4 \\
\hline $\begin{array}{l}\text { Toki et al. (1998) } \\
\text { [11] }\end{array}$ & 64 & $\mathrm{D} \& \mathrm{C}$ & & 91 & & & 96 \\
\hline \multirow{2}{*}{$\begin{array}{l}\text { Wang and Guo } \\
\text { (2002) [12] }\end{array}$} & 156 & $\mathrm{D} \& \mathrm{C}$ & 70 & & & 85 & 34 \\
\hline & & $\mathrm{H}$ & 90 & & & 97 & 57 \\
\hline $\begin{array}{l}\text { Mencaglia et al. } \\
\text { (1984) [13] }\end{array}$ & 23 & $\mathrm{H}$ & 92 & & & & \\
\hline $\begin{array}{l}\text { Lo et al. (2001) } \\
\text { [14] }\end{array}$ & 200 & $\mathrm{H}$ & 92.5 & 68.3 & 98.7 & 93.3 & 92.4 \\
\hline \multirow{2}{*}{$\begin{array}{l}\text { Kietlinska et al. } \\
\text { (1998) [15] }\end{array}$} & 38 & $\mathrm{H}$ & 65.8 & 33.3 & 68.6 & 8.3 & \\
\hline & & US & 77.8 & 75 & 78 & & \\
\hline \multirow{2}{*}{$\begin{array}{l}\text { Gabrielli et al. } \\
(1996)[16]\end{array}$} & 67 & $\mathrm{H}$ & 72 & 64 & 73 & 32 & 91 \\
\hline & & US & 82 & 54 & 87 & 46 & 91 \\
\hline $\begin{array}{l}\text { Kose et al. } \\
\text { (2003) [17] }\end{array}$ & 43 & US & 97.8 & 75.0 & 100 & & \\
\hline \multirow[t]{3}{*}{ Present study } & 52 & $\mathrm{H}$ & 76.9 & 42.9 & 89.5 & 60.0 & 81.0 \\
\hline & 75 & $\mathrm{D} \& \mathrm{C}$ & 64.0 & 57.9 & 66.1 & 36.7 & 82.2 \\
\hline & & US & 76.7 & 15.8 & 98.1 & 75.0 & 76.8 \\
\hline
\end{tabular}

In their study, the sensitivity was dependent on the degree of cervical infiltration. With involvement of the cervical glands alone, the sensitivity was $50 \%$, while with involvement of the cervical stroma alone the figure was $0 \%$. Infiltration of both the cervical glands and the stroma produced a sensitivity of $86.2 \%$. The results for hysteroscopy and macroscopic assessment were almost identical. Considerably more negative results for hysteroscopy in 38 patients were reported by Kietlinska et al. in a study comparing fractional curettage, hysteroscopy, and imaging procedures such as ultrasonography, computed tomography, and magnetic resonance imaging [15]. For hysteroscopy, the diagnostic accuracy for cervical involvement was given as $65.8 \%$, with a sensitivity of $33.3 \%$, a specificity of $68.6 \%$, and a positive predictive value of $8.3 \%$. In a study including 67 patients in which transvaginal ultrasonography was compared with hysteroscopy in the assessment of cervical involvement in endometrial carcinoma, Gabrielli et al. in 1996 [16] obtained similarly poor results for hysteroscopy. Hysteroscopy was found to have an accuracy of $72 \%$ for the diagnosis of cervical involvement. The sensitivity was $64 \%$, the specificity $73 \%$, the positive predictive value $32 \%$, and the negative predictive value 91\%. Overall, hysteroscopy and transvaginal ultrasonography produced comparable results. Our own data for hysteroscopy are comparable with those of Kietlinska et al. [15] and Gabrielli et al. [16], with an accuracy rate for diagnosing or excluding cervical involvement of $76.9 \%$, a sensitivity of $42.9 \%$, a specificity of $89.5 \%$, a positive predictive value of $60 \%$, and a negative predictive value of $81.0 \%$. As in the study by Lo et al. [14], the present data also showed a difference in the sensitivity of hysteroscopy relative to the degree of cervical infiltration. In our examinations, the sensitivity for infiltration of the cervical glands was $20 \%$, while for infiltration of the cervical glands and cervical stroma it was $55.6 \%$.

In our examinations, ultrasonography provided an extremely poor sensitivity figure of $15.8 \%$ for assessing cervical involvement. This would at best make it possible to diagnose cervical involvement, but not to exclude it. The specificity was $98.1 \%$, and the accuracy for excluding or demonstrating cervical infiltration was $76.7 \%$. This might have been due to the fact that during the ultrasound examination, greater importance was attached to the depth of myometrial infiltration than to cervical infiltration. Gabrielli et al. also considered that transvaginal ultrasonography is only of limited value for assessing cervical involvement [16]. They calculated an accuracy of $82 \%$, a sensitivity of $54 \%$, and a specificity of $87 \%$. In contrast to these findings, Kose et al. report an accuracy rate of $97.8 \%$, a sensitivity of $75.0 \%$, and a specificity of $100 \%$ [17]. However, their study only included a total of 43 patients, four of whom $(9.3 \%)$ had histological confirmation of cervical involvement. 
We would conclude from the data obtained in the present study that each individual procedure on its own is not adequate for pretreatment assessment of intracavitary tumor spread in endometrial carcinoma. However, if endocervical curettage is negative and hysteroscopy shows no cervical infiltration, it often may be possible to identify patients correctly who are at a lower risk, i.e. those without cervical involvement. Because hysteroscopy and fractional curettage feature relatively high negative predictive values of $81.0 \%$ and $82.2 \%$. In this way, an overtreatment could be prevented in $91.8 \%$ of patients (56 of 61 ) without involvement of the cervical stroma. In particular, hysteroscopy is more sensitive in assessing cervical infiltration if it extends into the cervical stroma (55.6\%) than when there is infiltration of the cervical glands alone (20.0\%). However, the National Comprehensive Cancer Network ( $\mathrm{NCCN}$ ) only recommends clinically assuming FIGO stage IIB and carrying out radical hysterectomy if there is clear evidence of stromal infiltration in the cervical abrasion sample [3]. In the final analysis, the potential benefits of a high-risk operation such as radical hysterectomy may be relativized by the patient's age and concomitant diseases.

\section{References}

1. Foley K, Lee RB (1990) Surgical complications of obese patients with endometrial carcinoma. Gynecol Oncol 39:171-174

2. Tozzi R, Malur S, Koehler C, Schneider A (2005) Laparoscopy versus laparotomy in endometrial cancer: first analysis of survival of a randomized prospective study. J Minim Invas Gyn L 12:130-136

3. National Comprehensive Cancer Network (2006) Uterine cancers, version 1.2006. Clinical practice guidelines in oncology. Available at: http://www.nccn.org/professionals/physician_gls/PDF/uterine. pdf. Accessed 24 May 2007

4. Beckmann MW (2003) Überarbeitete S1-Leitlinien Endometriumkarzinom. Interdisziplinäre Organkommission Uterus der Arbeits- gemeinschaft für Gynäkologische Onkologie (AGO) in der DKG und DGGG. Onkologe 9:1262-1266

5. Emons G (2006) Endometrial cancer: suggestions for the update of the guidelines (in German). Zentralbl Gynäkol 128:18-22

6. Beckmann MW, Link M, Lux MP, Fasching PA, Ackermann S (2005) Early cancer detection in women (in German). Gynäkologe 38:349-363

7. Ackermann S, Beckmann MW (2005) Does cervical cancer therapy in Germany correlate with the German clinical practice guidelines? A survey by the Arbeitsgemeinschaft Gynäkologische Onkologie (AGO) (in German). Geburtshilfe Frauenheilkd 65:164-171

8. FIGO (1991) Annual report. Int J Gynecol Obstet 36(Suppl):38-39

9. Heyman J, Reuterwall O, Brenner S (1941) The Radiumhemmet experience with radiotherapy in cancer of the corpus of the uterus. Acta Radiol 22:14-98

10. Ayhan A, Yarali H, Urman B et al (1990) Comparison of clinical and surgical-pathologic staging in patients with endometrial carcinoma. J Surg Oncol 43:33-35

11. Toki T, Oka K, Nakayama K, Oguchi O, Fujii S (1998) A comparative study of pre-operative procedures to assess cervical invasion by endometrial carcinoma. Brit J Obstet Gynaec 105:512-516

12. Wang W, Guo Y (2002) Value of hysteroscopy and dilatation and curettage in diagnosis of endometrial carcinoma (in Chinese). Zhonghua Fu Chan Ke Za Zhi 37:550-552

13. Mencaglia L, Scarselli G, Tantini C (1984) Hysteroscopic evaluation of endometrial cancer. J Reprod Med 29:701-704

14. Lo KW, Cheung TH, Yim SF, Chung TK (2001) Preoperative hysteroscopic assessment of cervical invasion by endometrial carcinoma: a retrospective study. Gynecol Oncol 82:279-282

15. Kietlinska Z, Stelmachow J, Antczak-Judycka A, Timorek A, Sawicki W, Tyminska B (1998) Fractional curettage, hysteroscopy and imaging techniques: transvaginal sonography (TVS), magnetic resonance imaging (MRI) and computed tomography (CT) in the diagnosis of cervical canal involvement in cases of endometrial carcinoma. Eur J Gynaecol Oncol 19:561-564

16. Gabrielli S, Marabini A, Bevini M et al (1996) Transvaginal sonography vs. hysteroscopy in the preoperative staging of endometrial carcinoma. Ultrasound Obstet Gynecol 7:443-446

17. Kose G, Aka N, Api M (2003) Preoperative assessment of myometrial invasion and cervical involvement of endometrial cancer by transvaginal ultrasonography. Gynecol Obstet Inves $56: 70-76$ 